\title{
Numerical simulation of dynamic behavior of droplet on solid surface by the two-phase lattice Boltzmann method
}

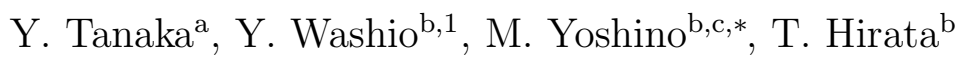 \\ ${ }^{a}$ Department of Mathematics and System Development Engineering, Interdisciplinary \\ Graduate School of Science and Technology, Shinshu University, 4-17-1, Wakasato, \\ Nagano-shi, Nagano 380-8553, Japan \\ ${ }^{b}$ Department of Mechanical Systems Engineering, Shinshu University, 4-17-1, Wakasato, \\ Nagano-shi, Nagano 380-8553, Japan \\ ${ }^{c}$ CREST, Japan Science and Technology Agency, 4-1-8, Honcho, Kawaguchi-shi, \\ Saitama 332-0012, Japan
}

\section{Abstract}

The dynamic behavior of a droplet on a solid surface is simulated by the lattice Boltzmann method (LBM) for two-phase fluids with large density differences; the wetting boundary condition on solid walls is incorporated in this simulation. By using the method, the dynamic behavior of a droplet impinging on a horizontal wall is investigated in terms of various Weber numbers. The dynamic contact angle, the contact line velocity, and the wet length are calculated, and found to be in good agreement with available experimental data. In addition, the method is applied to simulations of the collision of a falling droplet with a stationary droplet on a solid surface. The

\footnotetext{
*Corresponding author. Tel.:+81 26269 5116; fax:+81 262695116.

Email address: masato@shinshu-u.ac.jp (M. Yoshino)

${ }^{1}$ Present address: Seiko Epson Corporation, Suwa-shi, Nagano 392-8502, Japan.
} 
behavior of the droplets and the mixing process during their collision are simulated in terms of various impact velocities and several static contact angles on the solid surface. It is seen that mixing occurs around the rim of the coalescent droplet due to the circular flows. Also, the relationship between the mixing rate of the primary coalescent droplet and Weber number is investigated.

Keywords: Droplet, Wettability, Weber Number, Lattice Boltzmann Method (LBM), Mobility.

\section{Introduction}

The control of wettability on solid surfaces is an important issue in both physical and chemical research and many engineering fields. Recently, the attempt has been made to develop substrates with an innovative capability that exploits the macroscopic fluid properties such as surface tension and viscosity. For example, in the automotive industry, several coating techniques have been developed to control the wettability of solid surfaces. A hydrophobic coating is used for automobile windshields because it is effective in waterproofing, rustproofing, and suppressing ice formation on solid surfaces. A hydrophilic coating is used for rear-view mirrors of automobiles owing to its anti-fogging property. 
Wettability of solid surfaces has usually been evaluated by measuring the static contact angle of a droplet on a solid surface. However, it is necessary for the wettability to be evaluated dynamically when the droplet is moving on the surface (e.g., the spin-coating process and the removal process of droplets). Sikalo et al. [1] made an experimental quantitative investigation of the dynamic behavior of a droplet impinging on a horizontal surface. They measured the diameter of the wetting area on the solid surface and the apex height of the droplet, and studied the relationships between several properties such as liquid surface tension, fluid viscosity, contact angle, and surface roughness. Sikalo et al. [2] measured time variations of the contact angle (dynamic contact angle) for various impact velocities. From their experimental results, they concluded that the dynamic contact angle is a function of contact line speed and the flow characteristics in the vicinity of the moving contact line. Sikalo and Ganic [3] experimentally investigated the impact of a droplet on a shallow liquid layer and compared these results with those obtained for a droplet on a horizontal surface. In general, the space- and time-scales for these phenomena are very small; hence, numerical simulation is an effective approach to investigate the dynamics of a droplet on solid surfaces in detail. Earlier, Lunkad et al. [4] carried out simulations of the 
dynamic behavior of a droplet impinging on horizontal and inclined surfaces by the Volume of Fluid (VOF) method and examined the effects of wettability and the inclined angle on the diameter of a wetted spot. Manservisi and Scardovelli [5] proposed a finite element method coupled with the interface front-tracking method to investigate the motion of a droplet on horizontal surfaces with different wettability. Fujimoto et al. [6] also investigated the collision of a droplet with a hemispherical droplet on a solid surface by experimental and numerical approaches. From their results, it is found that a circular liquid crown appears due to a large pressure gradient near the free surface. Although several simulations have been conducted so far, few studies have focused on the effect of surface tension, viscous force, and inertia force on the motion of a droplet. Moreover, the collision of droplets on solid surfaces is not fully understood. These phenomena are observed in liquid microchannels; therefore, it is necessary to understand the dynamic behavior and mixing of droplets on solid surfaces.

Since the mid-1990s, the lattice Boltzmann method (LBM) for two-phase fluids has been regarded as a promising numerical scheme for two-phase fluids, and has been applied to many two-phase flow simulations $[7,8]$. In particular, Inamuro et al. [9] recently proposed a two-phase LBM based on 
the diffuse interface method (DIM), which enables the stable calculation of two-phase fluid flows with large density ratios of up to 1000 . This method has been used to simulate the dynamics of droplets on solid surfaces [10]. In addition, Yoshino and Mizutani [11] incorporated the boundary condition proposed by Briant et al. [12, 13], which is based on the wetting potential [14], into the abovementioned two-phase LBM. In the DIM, a parameter called mobility, which is related to the ease of coalescence of the interfaces, is considered. Inamuro et al. [9] pointed out that coalescence is sensitive to the mobility. Hence, similar consideration regarding the mobility is also needed for the interfaces when simulating droplet motion on a solid surface.

The aim of the present paper is to apply the LBM to the following two problems of microscale droplet motion. The first problem involves the investigation of the dynamic behavior of a droplet impinging on a horizontal surface in terms of various Weber numbers. The calculated results are evaluated by quantitative comparison with available experimental data. The second problem involves the investigation of the collision of a falling droplet with a stationary droplet on a solid surface. The behavior of both droplets and their mixing process during the collision are investigated in terms of various Weber numbers and several static contact angles on the solid surface. 


\section{Numerical method}

\subsection{Two-phase lattice Boltzmann method}

Non-dimensional variables, which are defined by using a characteristic length $\widetilde{L}$, a characteristic particle speed $\widetilde{c}$, a characteristic time scale $\widetilde{t}_{0}=$

$\widetilde{L} / \widetilde{V}\left(\widetilde{V}\right.$ : a characteristic flow speed), a reference order parameter $\widetilde{\phi}_{0}$, and a reference density $\widetilde{\rho}_{0}$, are used as in Ref. [9]. In the LBM, a modeled fluid, which is composed of identical particles whose velocities are restricted to a finite set of $N$ vectors $\boldsymbol{c}_{i}(i=1,2,3, \ldots, N)$, is considered. The nine-velocity model $(N=9)$ given by the following equation is used in the computations:

$$
\begin{aligned}
& {\left[\boldsymbol{c}_{1}, \boldsymbol{c}_{2}, \boldsymbol{c}_{3}, \boldsymbol{c}_{4}, \boldsymbol{c}_{5}, \boldsymbol{c}_{6}, \boldsymbol{c}_{7}, \boldsymbol{c}_{8}, \boldsymbol{c}_{9}\right]} \\
& =\left[\begin{array}{llllllllll}
0 & 1 & 0 & -1 & 0 & 1 & -1 & -1 & 1 \\
0 & 0 & 1 & 0 & -1 & 1 & 1 & -1 & -1
\end{array}\right]
\end{aligned}
$$

The physical space is divided into a square lattice, and the evolution of particle population at each lattice site is computed. Two particle velocity distribution functions, $f_{i}$ and $g_{i}$, are used. The function $f_{i}$ is used for the calculation of an order parameter which represents two phases, and the function $g_{i}$ is used for the calculation of a predicted velocity of the two-phase fluid without a pressure gradient. The evolution of the particle distribution 
functions $f_{i}(\boldsymbol{x}, t)$ and $g_{i}(\boldsymbol{x}, t)$ with velocity $\boldsymbol{c}_{i}$ at the point $\boldsymbol{x}$ and at time $t$ is computed by the following equations:

$$
\begin{aligned}
f_{i}\left(\boldsymbol{x}+\boldsymbol{c}_{i} \Delta x, t+\Delta t\right) & =f_{i}^{\mathrm{c}}(\boldsymbol{x}, t), \\
g_{i}\left(\boldsymbol{x}+\boldsymbol{c}_{i} \Delta x, t+\Delta t\right) & =g_{i}^{\mathrm{c}}(\boldsymbol{x}, t),
\end{aligned}
$$

where $f_{i}^{\mathrm{c}}$ and $g_{i}^{\mathrm{c}}$, given below, are functions in which variables $\boldsymbol{x}$ and $t$ enter only through macroscopic variables and/or their derivatives, $\Delta x$ is a spacing of the square lattice, and $\Delta t$ is a time step during which the particles travel the lattice spacing.

The order parameter $\phi$ distinguishing two phases and the predicted velocity $\boldsymbol{u}^{*}$ of the two-phase fluids are defined as follows:

$$
\begin{aligned}
\phi & =\sum_{i=1}^{9} f_{i}, \\
\boldsymbol{u}^{*} & =\sum_{i=1}^{9} \boldsymbol{c}_{i} g_{i} .
\end{aligned}
$$

The functions $f_{i}^{\mathrm{c}}$ and $g_{i}^{\mathrm{c}}$ in the Eqs. (2) and (3) are given as follows:

$$
f_{i}^{\mathrm{c}}=H_{i} \phi+F_{i}\left[p_{0}-\kappa_{f} \phi \frac{\partial^{2} \phi}{\partial x_{\alpha}^{2}}\right]
$$




$$
\begin{aligned}
& +3 E_{i} \phi c_{i \alpha} u_{\alpha}+E_{i} \kappa_{f} G_{\alpha \beta}(\phi) c_{i \alpha} c_{i \beta}+E_{i} C \frac{\partial P_{\alpha \beta}}{\partial x_{\beta}} c_{i \alpha} \\
g_{i}^{\mathrm{c}}= & E_{i}\left[1+3 c_{i \alpha} u_{\alpha}-\frac{3}{2} u_{\alpha} u_{\alpha}+\frac{9}{2} c_{i \alpha} c_{i \beta} u_{\alpha} u_{\beta}+\frac{3}{4} \Delta x\left(\frac{\partial u_{\beta}}{\partial x_{\alpha}}+\frac{\partial u_{\alpha}}{\partial x_{\beta}}\right) c_{i \alpha} c_{i \beta}\right. \\
& \left.+3 c_{i \alpha} \frac{\Delta x}{\rho} \frac{\partial}{\partial x_{\beta}}\left\{\mu\left(\frac{\partial u_{\beta}}{\partial x_{\alpha}}+\frac{\partial u_{\alpha}}{\partial x_{\beta}}\right)\right\}\right]+E_{i} \frac{\kappa_{g}}{\rho} G_{\alpha \beta}(\rho) c_{i \alpha} c_{i \beta} \\
& -\frac{1}{2} F_{i} \frac{\kappa_{g}}{\rho}\left(\frac{\partial \rho}{\partial x_{\alpha}}\right)^{2}-3 E_{i} c_{i y}\left(1-\frac{\rho_{\mathrm{G}}}{\rho}\right) g \Delta x
\end{aligned}
$$

where

$$
\begin{aligned}
p_{0} & =\frac{\phi T}{1-b \phi}-a \phi^{2} \\
G_{\alpha \beta}(\phi) & =\frac{9}{2} \frac{\partial \phi}{\partial x_{\alpha}} \frac{\partial \phi}{\partial x_{\beta}}-\frac{9}{4} \frac{\partial \phi}{\partial x_{\gamma}} \frac{\partial \phi}{\partial x_{\gamma}} \delta_{\alpha \beta} \\
P_{\alpha \beta} & =\left[p_{0}-\kappa_{f} \phi \frac{\partial^{2} \phi}{\partial x_{\gamma}^{2}}-\frac{\kappa_{f}}{2}\left(\frac{\partial \phi}{\partial x_{\gamma}}\right)^{2}\right] \delta_{\alpha \beta}+\kappa_{f} \frac{\partial \phi}{\partial x_{\alpha}} \frac{\partial \phi}{\partial x_{\beta}}
\end{aligned}
$$

and

$$
\begin{aligned}
& H_{1}=1, H_{2}=H_{3}=\cdots=H_{9}=0, \\
& F_{1}=-5 / 3, F_{i}=3 E_{i}(i=2,3, \ldots, 9), \\
& E_{1}=4 / 9, E_{2}=E_{3}=E_{4}=E_{5}=1 / 9, E_{6}=E_{7}=E_{8}=E_{9}=1 / 36 .
\end{aligned}
$$

In the above equations, $\alpha, \beta, \gamma=x, y$ (subscripts $\alpha, \beta$, and $\gamma$ represent Cartesian coordinates and the summation convention is used), $\delta_{\alpha \beta}$ is the Kronecker delta, and subscripts $\mathrm{L}$ and $\mathrm{G}$ indicate respective liquid and gas phases. $\boldsymbol{u}$, 
$\rho$, and $\mu$ are the velocity, the density, and the viscosity of two-phase fluids, respectively. $g$ is the gravitational acceleration which is acting in the $-y$ direction. $\kappa_{f}$ and $\kappa_{g}$ are constant parameters determining the width of the interface and the strength of the interfacial tension $\sigma$, respectively. $a, b$, and $T$ are free parameters determining the maximum and minimum values of $\phi$, and $C$ is a constant parameter related to the mobility $\theta_{\mathrm{M}}$ as follows:

$$
\theta_{\mathrm{M}}=\left(\frac{1}{2}-\frac{1}{3} C\right) \Delta x
$$

The first and second derivatives in Eqs. (6), (7), (9), and (10) are calculated by using the following second-order finite difference approximations:

$$
\begin{aligned}
\frac{\partial \psi}{\partial x_{\alpha}} & =\frac{1}{6 \Delta x} \sum_{i=2}^{9} c_{i \alpha} \psi\left(\boldsymbol{x}+\boldsymbol{c}_{i} \Delta x\right) \\
\frac{\partial^{2} \psi}{\partial x_{\alpha}^{2}} & =\frac{1}{3(\Delta x)^{2}}\left[\sum_{i=2}^{9} \psi\left(\boldsymbol{x}+\boldsymbol{c}_{i} \Delta x\right)-8 \psi(\boldsymbol{x})\right] .
\end{aligned}
$$

The density in the interface is obtained by using the cut-off values of the order parameter, $\phi_{\mathrm{L}}^{*}$ and $\phi_{\mathrm{G}}^{*}$, for the liquid and gas phases with the following 
relation:

$$
\rho= \begin{cases}\rho_{\mathrm{G}}, & \phi<\phi_{\mathrm{G}}^{*} \\ \frac{\rho_{\mathrm{L}}-\rho_{\mathrm{G}}}{2}\left\{\sin \left[\frac{\phi-\left(\phi_{\mathrm{L}}^{*}+\phi_{\mathrm{G}}^{*}\right) / 2}{\phi_{\mathrm{L}}^{*}-\phi_{\mathrm{G}}^{*}}\right]+1\right\}+\rho_{\mathrm{G}}, & \phi_{\mathrm{G}}^{*} \leq \phi \leq \phi_{\mathrm{L}}^{*} . \\ \rho_{\mathrm{L}}, & \phi_{\mathrm{L}}^{*}<\phi\end{cases}
$$

The viscosity $\mu$ in the interface is obtained by the following equation:

$$
\mu=\frac{\rho-\rho_{\mathrm{G}}}{\rho_{\mathrm{L}}-\rho_{\mathrm{G}}}\left(\mu_{\mathrm{L}}-\mu_{\mathrm{G}}\right)+\mu_{\mathrm{G}}
$$

The interfacial tension $\sigma$ is given by

$$
\sigma=\kappa_{g} \int_{-\infty}^{\infty}\left(\frac{\partial \rho}{\partial \xi}\right)^{2} d \xi
$$

where $\xi$ indicates the coordinate normal to the interface [15].

Since $\boldsymbol{u}^{*}$ does not satisfy the continuity equation in general, it is required for $\boldsymbol{u}^{*}$ to be corrected by using the Poisson equation for the pressure $p$ of the two-phase fluids. Hence, the velocity distribution function $h_{i}$ is introduced, and the following evolution equation of $h_{i}$ is used for the calculation of $p$ :

$$
h_{i}^{n+1}\left(\boldsymbol{x}+\boldsymbol{c}_{i} \Delta x\right)=h_{i}^{n}(\boldsymbol{x})-\frac{1}{\tau_{h}}\left[h_{i}^{n}(\boldsymbol{x})-E_{i} p^{n}(\boldsymbol{x})\right]-\frac{1}{3} E_{i} \frac{\partial u_{\alpha}^{*}}{\partial x_{\alpha}} \Delta x
$$


where $n$ is the number of iterations and the relaxation time $\tau_{h}$ is give by

$$
\tau_{h}=\frac{1}{\rho}+\frac{1}{2}
$$

The pressure $p$ is obtained by

$$
p=\sum_{i=1}^{9} h_{i} .
$$

The calculation of Eq. (18) is iterated until $\left|p^{n+1}-p^{n}\right| / \rho<\varepsilon$ is satisfied in the whole domain. The tolerance $\varepsilon$ is set to $1.0 \times 10^{-6}$ in the following calculations.

Applying the asymptotic theory [16] to Eqs. (2), (3), and (18), we find that the asymptotic expansions of macroscopic variables, $\phi, \rho, \boldsymbol{u}$, and $p$, satisfy the phase-field advection-diffusion equation (the Cahn-Hilliard equation with advection) for $\phi$, the continuity equation, and the Navier-Stokes equations for incompressible two-phase fluid including the interfacial tension and the buoyancy with relative errors of $O\left[(\Delta x)^{2}\right][17]$. 


\subsection{Wetting boundary condition}

Recently, Briant et al. [12, 13] have proposed a wetting boundary condition which enables the contact angle of the interface to be controlled in a way consistent with Cahn theory [14]. In their method, the derivative of the density normal to the wall, $\partial \rho / \partial n$, is specified by using the wetting potential that is calculated according to a prescribed static contact angle $\theta_{\mathrm{S}}$. In the following computations, the specified value of $\partial \rho / \partial n$ at wall sites is substituted for the first and second derivatives in Eq. (6).

\section{Results and discussion}

\subsection{A droplet impinging on horizontal surface}

The dynamic behavior of a droplet impinging on a horizontal surface is investigated. According to previous studies such as Ref. [18], two-dimensional computational results of the impingement of liquid drops on dry walls agree with the available experimental results [19]. Therefore, these phenomena are assumed to be two-dimensional in the following simulations. As shown in Fig. 1, a rectangular domain with $L_{x} \times L_{y}=450 \Delta x \times 300 \Delta x$ is considered. A liquid droplet with diameter $D=100 \Delta x$ is placed in contact with the bottom wall $(y=0)$, and it collides with the surface with an im- 
pact velocity $V$ at an initial time $(t=0)$. In the simulations, the wetting boundary condition is applied to the bottom wall, and the static contact angle is set to $\theta_{\mathrm{S}}=\pi / 2$. It should be noted that henceforth the static and dynamic contact angles are defined as dimensional quantities with radian unit. At the top boundary $\left(y=L_{y}\right)$, the normal derivatives of the order parameter, of the density, and of the velocity are set to zero. The periodic boundary condition is used for the other boundaries of the domain. The parameters determining the maximum and minimum values of the order parameter are $a=1, b=6.7, T=3.5 \times 10^{-2}$; it follows that these values are $\phi_{\max }=9.714 \times 10^{-2}$ and $\phi_{\min }=1.134 \times 10^{-2}$, respectively. The cut-off values of the order parameter for obtaining the density in the interface are $\phi_{\mathrm{L}}^{*}=9.2 \times 10^{-2}$ and $\phi_{\mathrm{G}}^{*}=1.5 \times 10^{-2}$. The other computational parameters are as follows: $\rho_{\mathrm{L}}=50, \rho_{\mathrm{G}}=1, \mu_{\mathrm{L}}=1.99 \Delta x, \mu_{\mathrm{G}}=3.08 \times 10^{-4} \Delta x$, $\kappa_{f}=1.40(\Delta x)^{2}, \kappa_{g}=1.27 \times 10^{-6}(\Delta x)^{2}$, and $g \Delta x=6.08 \times 10^{-11}$. The dimensionless numbers for this problem are Weber number $W e=\rho_{\mathrm{L}} V^{2} D / \sigma$ and Reynolds number $R e=\rho_{\mathrm{L}} V D / \mu_{\mathrm{L}}$.

First of all, in order to investigate the effect of mobility on the droplet behavior, the constant parameter $C$ is changed so that $\theta_{\mathrm{M}}$ can become a value between $0.005 \Delta x$ and $0.5 \Delta x$. The impact velocity is set to $V=2.35 \times 10^{-3}$. 
The Weber and Reynolds numbers become $W e=51$ and $R e=5.9$. As shown in Fig. 2, the computational results are compared with the theoretical prediction [20] in terms of the relationship between the dynamic contact angle $\theta_{\mathrm{D}}$ and capillary number $C a=U \mu_{\mathrm{L}} / \sigma$, where $\theta_{\mathrm{D}}$ and the contact line velocity $U$ are defined in Fig. 3. From Fig. 2, it is found that for lower capillary numbers $(C a<0.1), \theta_{\mathrm{D}}$ approaches the theoretical prediction as the mobility decreases. Thus, $\theta_{\mathrm{M}}=0.005 \Delta x$ is appropriate for the behavior of a droplet on the solid surface, and the value is used in the following simulations.

Next, the simulations are carried out by changing the impact velocity $V$ in the range of $50.8 \leq W e \leq 802$ and $5.90 \leq R e \leq 23.5$. Figs. 4 and 5 show the computational results of the dynamic contact angle $\theta_{\mathrm{D}}$, the wet length $d$, and the contact line velocity $U$ for $W e=51$ and 802 , respectively. In these figures, the results of the corresponding experiments performed by Sikalo et al. [2] are also shown. Note that, in order to compare the two results, the computed results are converted into dimensional quantities by using the aforementioned characteristic or reference values described in Sec. 2. In both figures, the wet length increases monotonously and finally approaches an equilibrium value. The contact line velocity, on the contrary, decreases with time and finally becomes zero. The dynamic contact angle is 
kept constant for some time after impact, but decreases rapidly and finally approaches the prescribed static contact angle. The main difference between the results for $W e=51$ and 802 is that the wet length for $W e=802$ decreases after $\widetilde{t} \widetilde{V} / \widetilde{D} \approx 10$ because of the inertial effect, while the wet length for $W e=51$ remains constant. These calculated results for both $W e=51$ and 802 agree well with the experimental data; the experiments were performed using a glycerin droplet with diameter $\widetilde{D}=2.45 \mathrm{~mm}(W e=51$ and $R e=27)$. Also, the static contact angle $\widetilde{\theta}_{\mathrm{S}}[\mathrm{rad}]$ on the solid surface was a certain angle between $0.50 \pi$ and $0.54 \pi$. Note that there is a difference between the experiments and the simulations: for real glycerin-air systems, the density ratio is about 1000 at standard temperature and pressure, while it is set to 50 in the simulations. In the present study, it is assumed that the density ratio is less effective and the surface tension is the predominant property, because the scale of length is so small in such flow problems. Hence, the Weber number is the dominant parameter for these phenomena, though detailed investigation concerning the effect of the density ratio is required in future work. In the preliminary computations, as the mobility increases (e.g., for $\theta_{\mathrm{M}}=0.5 \Delta x$ ), similar results are obtained for the wet length and the contact line velocity for different $\theta_{\mathrm{M}}$, but the calculated dynamic contact 
angle deviates from the experimental data, especially when the dynamic contact angle begins to decrease at an earlier time. This is because the diffusion flux in the interfaces is overestimated.

Figs. 6 and 7 show the time variations of $d / D$ and $h / D$, respectively, for various Weber numbers. For each Weber number, the value of $d / D$ increases with time and reaches a maximum value; the maximum value becomes larger as the Weber number increases. Then, the value of $d / D$ decreases and approaches the same constant value of $d / D=1.35$ in all cases. The time variation of $h / D$ is different from that of $d / D$, and the constant value in the equilibrium state is $h / D=0.704$. Considering the Weber number indicates the ratio of the inertia and interfacial forces, it is natural that the droplet should become deformable along the solid surface as the Weber number increases. Moreover, it is expected that the droplet reaches the equilibrium state due to the balance between the viscous force and interfacial force under the constant gravity. Because the capillary length $l=\sqrt{\sigma /\left(\rho_{\mathrm{L}} g\right)}$, which is related to the thickness of the liquid film [21], is the same value in all cases for a fixed static contact angle and for a constant initial diameter of the liquid droplet, it is considered that $d / D$ and $h / D$ approach their respective equilibrium values, regardless of different Weber numbers. 


\subsection{Droplet collision on solid surface}

The collision dynamics of a falling droplet with a stationary droplet on a solid surface, as shown in Fig. 8, is investigated. The computational domain is $L_{x} \times L_{y}=1500 \Delta x \times 450 \Delta x$. A stationary droplet with diameter $D=$ $100 \Delta x$ is placed on the bottom wall under the gravity and left until it reaches an equilibrium state. Then, another falling droplet with equal diameter $D=100 \Delta x$ collides with an impact velocity $V$ at an initial time $(t=0)$. The boundary conditions are the same as those in the previous problem. The computational parameters are $\rho_{\mathrm{L}}=50, \rho_{\mathrm{G}}=1, \mu_{\mathrm{L}}=2.27 \times 10^{-2} \Delta x$, $\mu_{\mathrm{G}}=4.08 \times 10^{-4} \Delta x, \kappa_{f}=1.40(\Delta x)^{2}, \kappa_{g}=2.61 \times 10^{-6}(\Delta x)^{2}, g \Delta x=$ $1.07 \times 10^{-10}, V=3.32 \times 10^{-3}$, and $\theta_{\mathrm{M}}=0.005 \Delta x$. The Weber and Reynolds numbers are then 50 and 731, respectively. As an example, if a water droplet with diameter $\widetilde{D}=2.5 \mathrm{~mm}$ impinges on a stationary droplet with velocity $\widetilde{V}=1.21 \mathrm{~m} / \mathrm{s}$ in the air at $293 \mathrm{~K}$, the dimensionless numbers are $W e=50$ and $R e=3010$. Fig. 9 shows the time evolution of the droplet shape and velocity vectors for $W e=50$ and $\theta_{\mathrm{S}}=\pi / 2$. Note that only the lower half of the whole domain $\left(0 \leq y \leq L_{y} / 2\right)$ is shown in Fig. 9 and in the following

Figs. 10-14. It can be seen that rims form after impact and circular flows appear around the rims. At $t^{*}=5.98 \times 10^{-1}$, where the dimensionless time 
$t^{*}$ is defined by $t V / D$, the maximum velocity is $4.12 \times 10^{-3}$ in the rims, which is comparable to the impact velocity. Then, the coalescent droplet spreads along the solid surface as the rims grow, and the circular flows move with the rims. Moreover, the droplet breaks up near the contact line at $t^{*}=2.59$. In order to investigate the mixing process of the droplets during collision, about 1800 black and 1800 gray tracer particles are initially embedded in the stationary and the falling droplets, respectively, and their locations are calculated by the fourth-order Runge-Kutta method. The locations of these particles are calculated every 200th time-step. Note that the particles going out of the droplets due to numerical errors are omitted in the calculations. Fig. 10 shows the calculated results of the time evolution of these tracer particles. It is seen that at $t^{*}=5.98 \times 10^{-1}$, both black and gray particles occupy the rims, and then only the black particles occupy the tip of each rim at $t^{*}=1.26$. After that, the coalescent droplet spreads with little mixing until $t^{*}=1.93$, but mixing is enhanced near the contact line while the rims settle down for $t^{*}>1.93$. In addition, the detached droplets almost entirely consist of the stationary droplet at $t^{*}=2.59$.

Next, in order to investigate the effect of the wettability on the solid surface, the droplet collisions on the hydrophilic wall $\left(\theta_{\mathrm{S}}=\pi / 3\right)$ and on 
the hydrophobic wall $\left(\theta_{\mathrm{S}}=2 \pi / 3\right)$ are simulated for $W e=50$. Figs. 11 and 12 show the time evolutions of the tracer particles for $\theta_{\mathrm{S}}=\pi / 3$ and $2 \pi / 3$, respectively. From Fig. 11 , it is seen that at $t^{*}=2.01$, the rims are elongated upward like a crown, which is a characteristic shape observed when a droplet impacts into a liquid film [22]. For $t^{*}>3.00$, the mixing of the droplets is accelerated by the falling rims, which cause the coalescent droplet to expand in the horizontal direction. Moreover, no splash is observed around the contact line in this case because of the hydrophilicity on the wall. On the other hand, in the case of the hydrophobic wall (Fig. 12), the rims are elongated laterally at $t^{*}=1.00$ owing to the rounded shape of the stationary droplet in the initial equilibrium state. Also, splashes do occur around the contact line of both the sides at $t^{*}=4.00$. Here, the mixing rate is defined by the percentage of the number of the gray particles in the total number of particles in the target droplet. The mixing rate of the detached droplets obtained in this case is $37.0 \%$. Also, the size of the detached droplets is larger than that for $W e=50$. After splashing, the spreading droplet contracts toward the center while the mixing still continues near the contact line. From these results, it is found that the collision characteristics are quite different according to wettability on the solid surface. 
Finally, simulations are carried out for $10 \leq W e \leq 100$ with fixed $\theta_{\mathrm{S}}=$ $\pi / 2$. The computational parameters are the same as those for $W e=50$ and $\theta_{\mathrm{S}}=\pi / 2$, except for the value of the impact velocity $V$. Figs. 13 and 14 show the time evolution of the tracer particles for $W e=10$ and 100 , respectively. From Fig. 13, it can be seen that at $t^{*}=8.20 \times 10^{-1}$, the rims are thicker than those for $W e=50$, and the coalescent droplet spreads without splashing in this case. For $W e=100$, on the other hand, the thickness of the rim is much smaller than that for $W e=50$. At $t^{*}=3.85$, the coalescent droplet breaks up and the detached droplets consist of only the stationary droplet. After that, the coalescent droplet, called the primary droplet, breaks up again at $t^{*}=7.52$. At this time, the detached droplets consist of the falling droplet exclusively. From Figs. 10, 13, and 14, it is seen that mixing occurs around the rim due to the circular flows. It is also found that the coalescent droplet breaks into smaller droplets for $W e=50$ and 100, while such a breakup does not occur for $W e=10$. Furthermore, the mixing rate of the primary droplet is quantitatively investigated. The relationship between the mixing rate of the primary droplet and Weber number is shown in Fig. 15. From this figure, it is found that the mixing rate increases for $W e<40$ and keeps almost constant for $40 \leq W e \leq 100$; in other words, the stationary and 
falling droplets splash at an almost constant ratio for $40 \leq W e \leq 100$.

\section{Concluding remarks}

We have applied the lattice Boltzmann method for two-phase fluids with large density differences, in which the wetting boundary condition was incorporated, to simulations of the dynamic behavior of a droplet on a solid surface. For a droplet impinging on a horizontal surface, the numerical results for a small mobility $\left(\theta_{\mathrm{M}}=0.005 \Delta x\right)$ agree with the available experimental data. For the collision of a falling droplet with a stationary droplet on a solid surface, mixing occurs around the rims due to the circular flows. With regard to the effect of wettability, collision characteristics are quite different in the hydrophilic and hydrophobic cases. As the static contact angle becomes larger, the direction in which the rims are elongated leans toward the horizontal direction and the size of the splashing droplet increases. In addition, with regard to the effect of Weber number, the coalescent droplet breaks into smaller droplets for $W e=50$ and 100, whereas the breakup of the coalescent droplet does not occur for $W e=10$. Also, the mixing rate of the primary droplet increases for $W e<40$ and keeps almost constant for $40 \leq W e \leq 100$. 
Finally, three-dimensional simulations are required in order to investigate other impact parameters (e.g., the position of the impact point and the impact angle). We are currently trying to perform three-dimensional simulations of droplet behavior on a solid surface. In addition, the simulation for higher density ratios is of importance in future work.

\section{Acknowledgment}

The authors thank the anonymous reviewers for critical comments on the manuscript.

\section{References}

[1] Sikalo S, Marengo M, Tropea C, Ganic EN. Analysis of impact of droplets on horizontal surfaces. Exp Therm Fluid Sci 2002;25:503-10.

[2] Sikalo S, Tropea C, Ganic EN. Dynamic wetting angle of a spreading droplet. Exp Therm Fluid Sci 2005;29:795-802.

[3] Sikalo S, Ganic EN. Phenomena of droplet-surface interactions. Exp Therm Fluid Sci 2006;31:97-110.

[4] Lunkad SF, Buwa VV, Nigam KDP. Numerical simulations of drop im- 
pact and spreading on horizontal and inclined surfaces. Chem Eng Sci $2007 ; 62: 7214-24$.

[5] Manservisi S, Scardovelli R. A variational approach to the contact angle dynamics of spreading droplets. Comput Fluids 2009;38:406-24.

[6] Fujimoto H, Ogino T, Takuda H, Hatta N. Collision of a droplet with a hemispherical static droplet on a solid. Int J Multiphase Flow 2001;27:1227-45.

[7] Chen S, Doolen GD. Lattice Boltzmann method for fluid flows. Ann Rev Fluid Mech 1998;30:329-64.

[8] Succi S. The lattice Boltzmann equation for fluid dynamics and beyond. Oxford University Press;2001.

[9] Inamuro T, Ogata T, Tajima S, Konishi N. A lattice Boltzmann method for incompressible two-phase flows with large density differences. J Comput Phys 2004;198:628-44.

[10] Yan YY, Zu YQ. A lattice Boltzmann method for incompressible twophase flows on partial wetting surface with large density ratio. J Comput Phys 2007;227:763-75. 
[11] Yoshino M, Mizutani Y. Lattice Boltzmann simulation of liquid-gas flows through solid bodies in a square duct. Math Comput Simulat 2006;72:264-9.

[12] Briant AJ, Papatzacos P, Yeomans JM. Lattice Boltzmann simulations of contact line motion in a liquid-gas system. Philos Trans R Soc Lond A 2002;360:485-95.

[13] Briant AJ, Wagner AJ, Yeomans JM. Lattice Boltzmann simulations of contact line motion. I. Liquid-gas systems. Phys Rev E 2004;69:031602.

[14] Cahn JW. Critical point wetting. J Chem Phys 1977;66:3667-72.

[15] Rowlinson JS, Widom B. Molecular theory of capillary. Clarendon Press Oxford;1982;50-68.

[16] Sone Y. Asymptotic theory of flow of rarefied gas over a smooth boundary II. In: Rarefied Gas Dynamics (ed. Dini D). Editrice Tecnico Scientifica;1971;2:737-49.

[17] Inamuro T. Lattice Boltzmann methods for viscous fluid flows and for two-phase fluid flows. Fluid Dyn Res 2006;38:641-59. 
[18] Mukherjee S, Abraham J. Investigation of drop impact on dry walls with a lattice-Boltzmann model. J Colloid Interface Sci 2007;312:341-54.

[19] Sikalo S, Wilhelm HD, Roisman IV, Jakirlic S, Tropea C. Dynamic contact angle of spreading droplets: Experiments and simulations. Phys Fluids 2005;17:062103.

[20] Jiang TS, Oh SG, Slattery JC. Correlation for dynamic contact angle. J Colloid Interface Sci 1979;69:74-7.

[21] de Gennes PG, Brochard-Wyart F. Quere D. Capillarity and Wetting Phenomena: Drops, Bubbles, Pearls, Waves. Springer;2004.

[22] Vander Wal RL, Berger GM, Mozes SD. Droplets splashing upon films of the same fluid of various depths. Exp Fluids 2006;40:33-52. 


\section{FIGURES}

Figure 1. Computational domain of a droplet impinging on horizontal surface.

Figure 2. Relationship between dynamic contact angle $\theta_{\mathrm{D}}$ and capillary number $C a$ for $W e=51$. The solid line indicates the theoretical prediction by Jiang et al. [20].

Figure 3. A droplet attached to horizontal surface: $\theta_{\mathrm{D}}$, dynamic contact angle; $U$, contact line velocity; $d$, wet length; $h$, height of droplet on the centerline.

Figure 4 . Time variations of dynamic contact angle, $\theta_{\mathrm{D}}$, contact line velocity, $U$, and wet length, $d / D$, for $W e=51$; (a) present results with $\theta_{\mathrm{M}}=0.005 \Delta x$; (b) experimental results by Sikalo et al. [2].

Figure 5. Time variations of dynamic contact angle, $\theta_{\mathrm{D}}$, contact line velocity, $U$, and wet length, $d / D$, for $W e=802$; (a) present results with $\theta_{\mathrm{M}}=0.005 \Delta x ;$ (b) experimental results by Sikalo et al. [2].

Figure 6. Time variation of $d / D$ for various Weber numbers.

Figure 7. Time variation of $h / D$ for various Weber numbers. 
Figure 8. Computational domain of collision of droplets on solid surface. The stationary droplet on the surface is in equilibrium.

Figure 9. Time evolution of droplet shape and velocity vectors for $W e=50$ and $\theta_{\mathrm{S}}=\pi / 2\left(t^{*}=t V / D\right)$. The lower half of the whole domain $(0 \leq y \leq$ $\left.L_{y} / 2\right)$ is shown.

Figure 10. Time evolution of tracer particles for $W e=50$ and $\theta_{\mathrm{S}}=\pi / 2$ $\left(t^{*}=t V / D\right)$. The lower half of the whole domain $\left(0 \leq y \leq L_{y} / 2\right)$ is shown.

Figure 11. Time evolution of tracer particles for $W e=50$ and $\theta_{\mathrm{S}}=\pi / 3$ $\left(t^{*}=t V / D\right)$. The lower half of the whole domain $\left(0 \leq y \leq L_{y} / 2\right)$ is shown.

Figure 12. Time evolution of tracer particles for $W e=50$ and $\theta_{\mathrm{S}}=2 \pi / 3$ $\left(t^{*}=t V / D\right)$. The lower half of the whole domain $\left(0 \leq y \leq L_{y} / 2\right)$ is shown.

Figure 13. Time evolution of tracer particles for $W e=10$ and $\theta_{\mathrm{S}}=\pi / 2$ $\left(t^{*}=t V / D\right)$. The lower half of the whole domain $\left(0 \leq y \leq L_{y} / 2\right)$ is shown.

Figure 14. Time evolution of tracer particles for $W e=100$ and $\theta_{\mathrm{S}}=\pi / 2$ $\left(t^{*}=t V / D\right)$. The lower half of the whole domain $\left(0 \leq y \leq L_{y} / 2\right)$ is shown.

Figure 15. Mixing rate of primary droplet versus Weber number. 


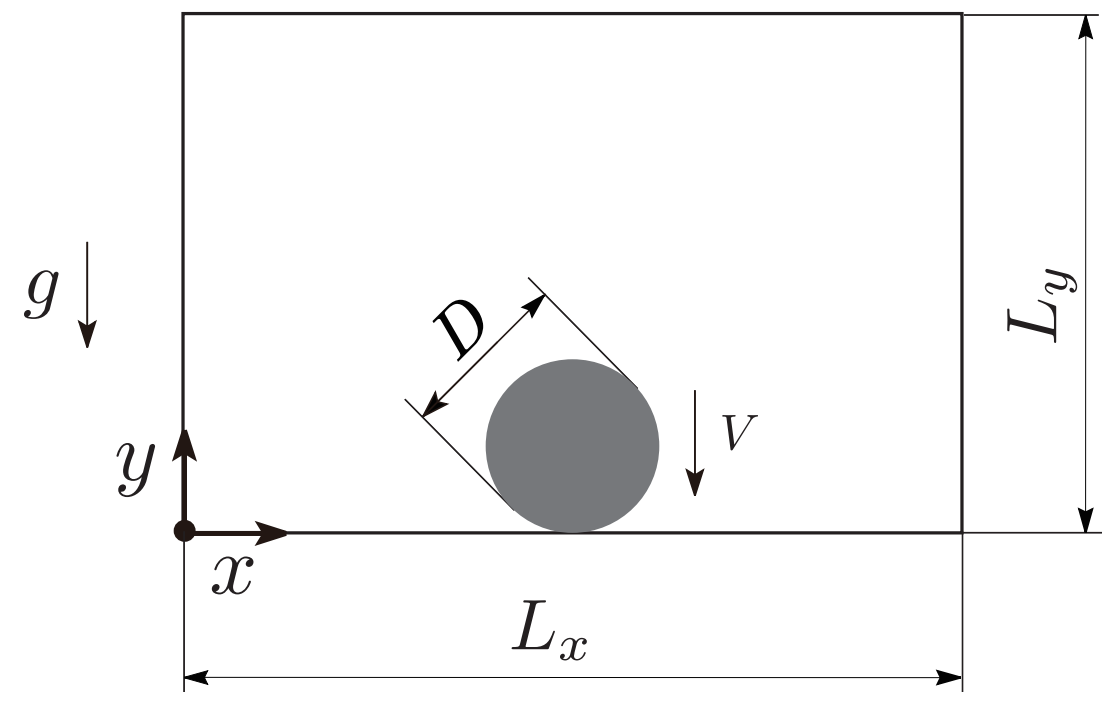

Figure 1: Computational domain of a droplet impinging on horizontal surface. 


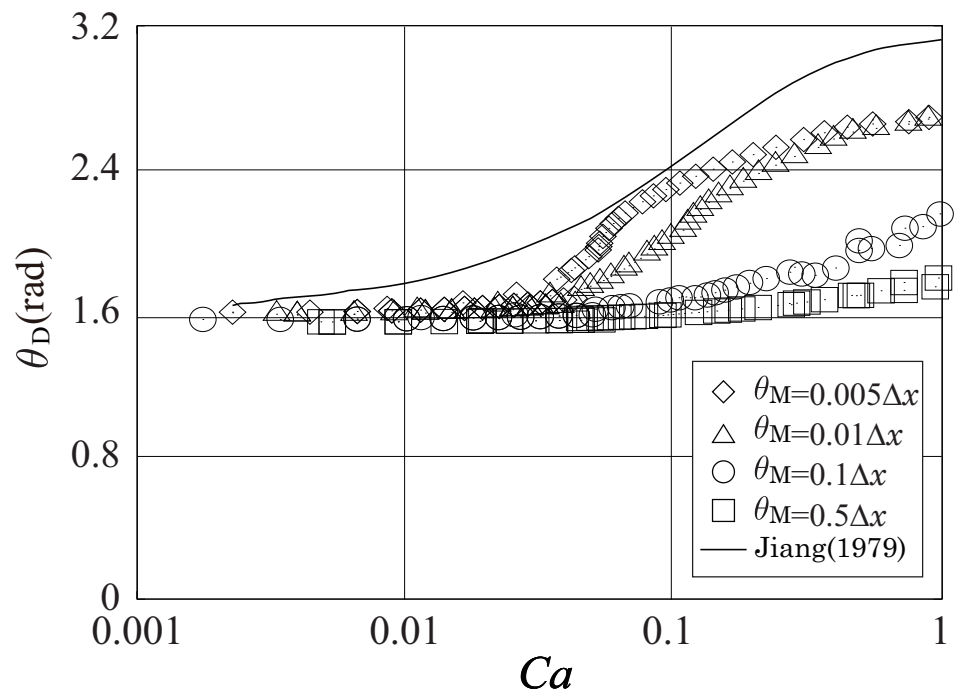

Figure 2: Relationship between dynamic contact angle $\theta_{\mathrm{D}}$ and capillary number $C a$ for $W e=51$. The solid line indicates the theoretical prediction by Jiang et al. [20]. 


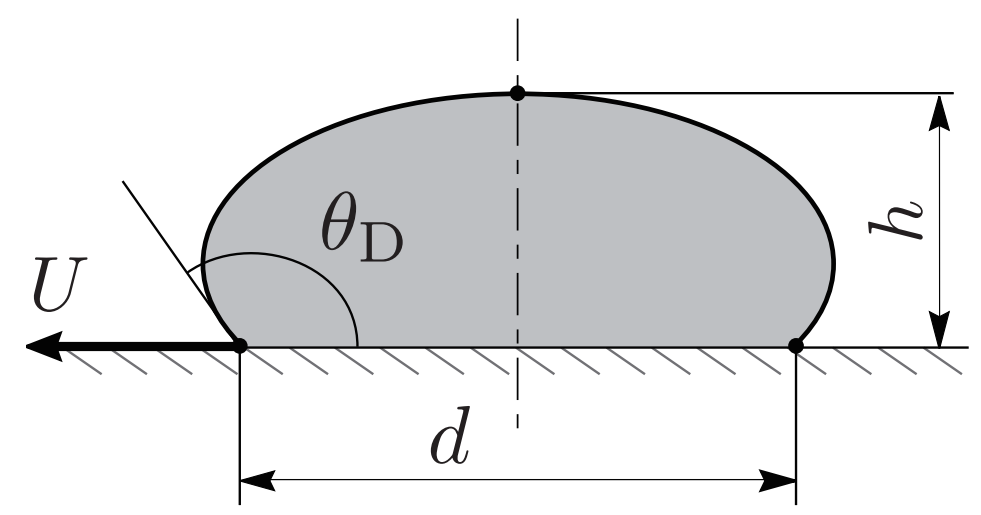

Figure 3: A droplet attached to horizontal surface: $\theta_{\mathrm{D}}$, dynamic contact angle; $U$, contact line velocity; $d$, wet length; $h$, height of droplet on the centerline. 


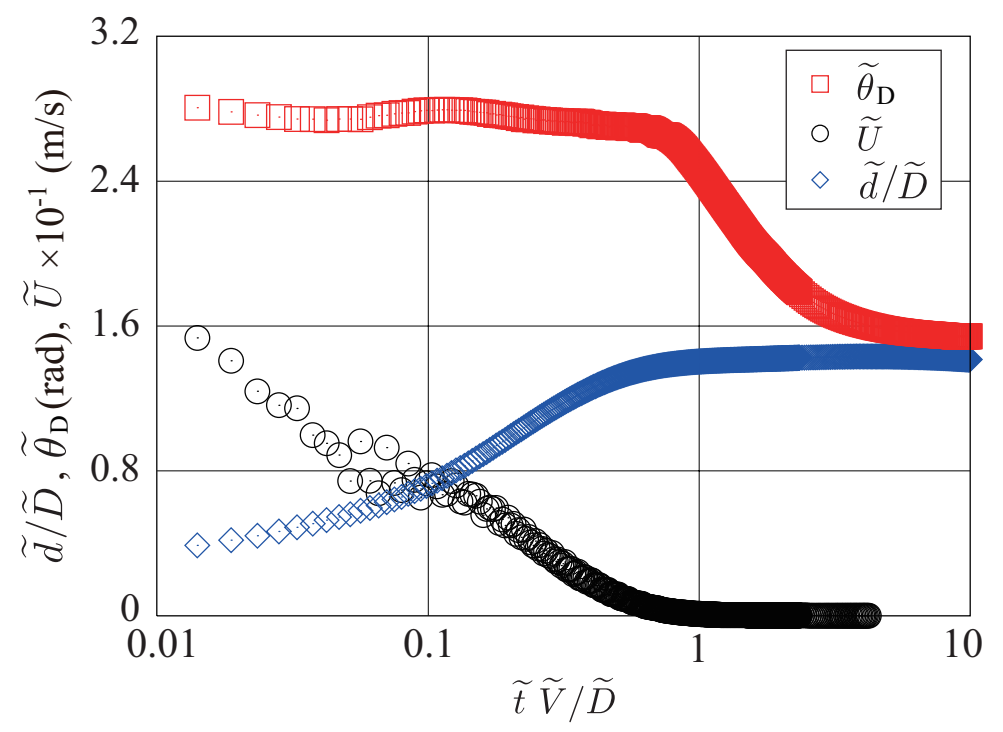

(a)

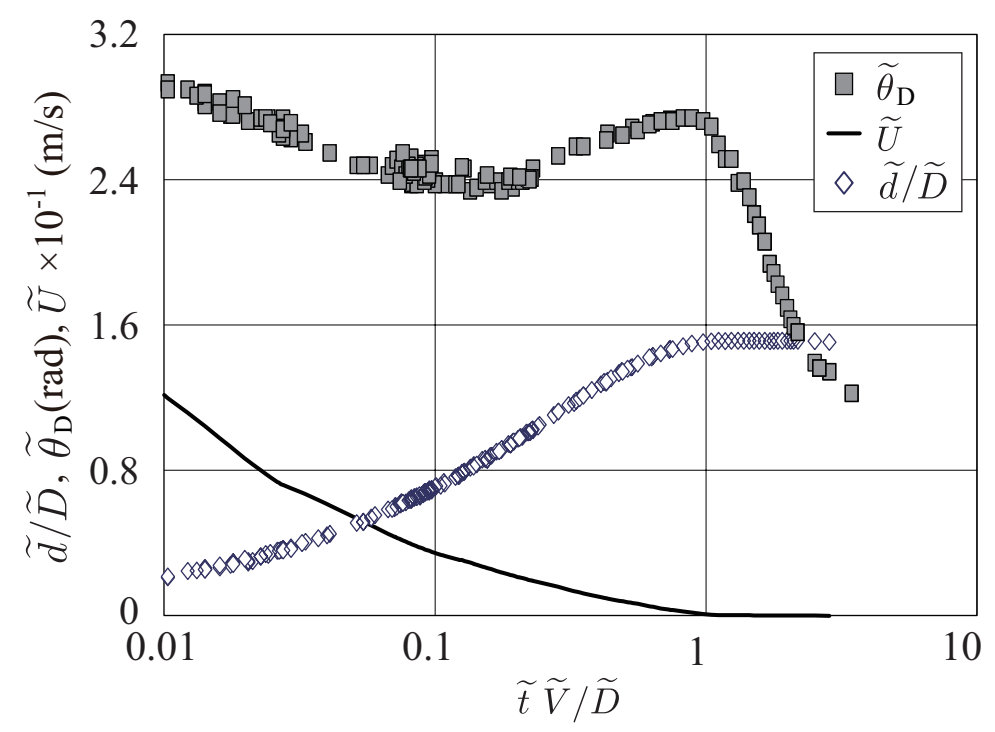

(b)

Figure 4: Time variations of dynamic contact angle, $\theta_{\mathrm{D}}$, contact line velocity, $U$, and wet length, $d / D$, for $W e=51$; (a) present results with $\theta_{\mathrm{M}}=0.005 \Delta x$; (b) experimental results by Sikalo et al. [2]. 


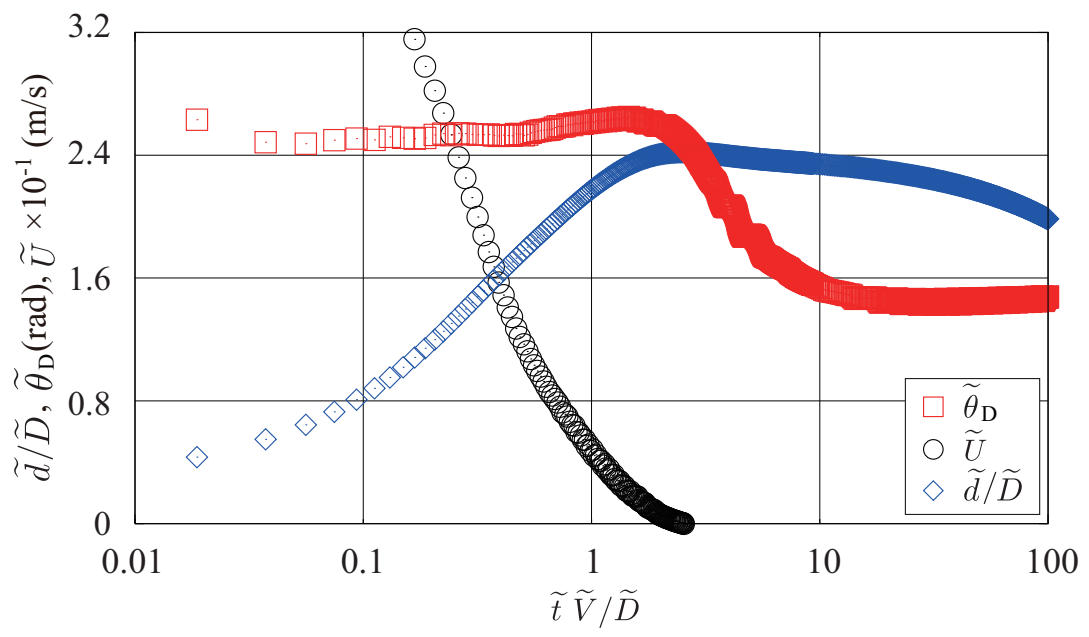

(a)

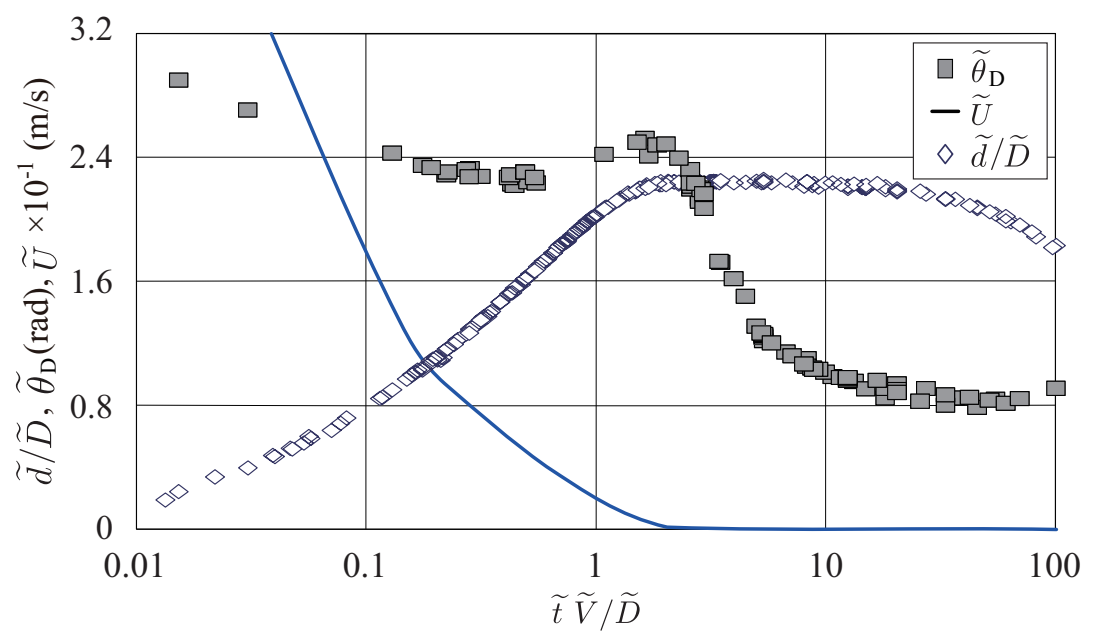

(b)

Figure 5: Time variations of dynamic contact angle, $\theta_{\mathrm{D}}$, contact line velocity, $U$, and wet length, $d / D$, for $W e=802$; (a) present results with $\theta_{\mathrm{M}}=0.005 \Delta x$; (b) experimental results by Sikalo et al. [2]. 


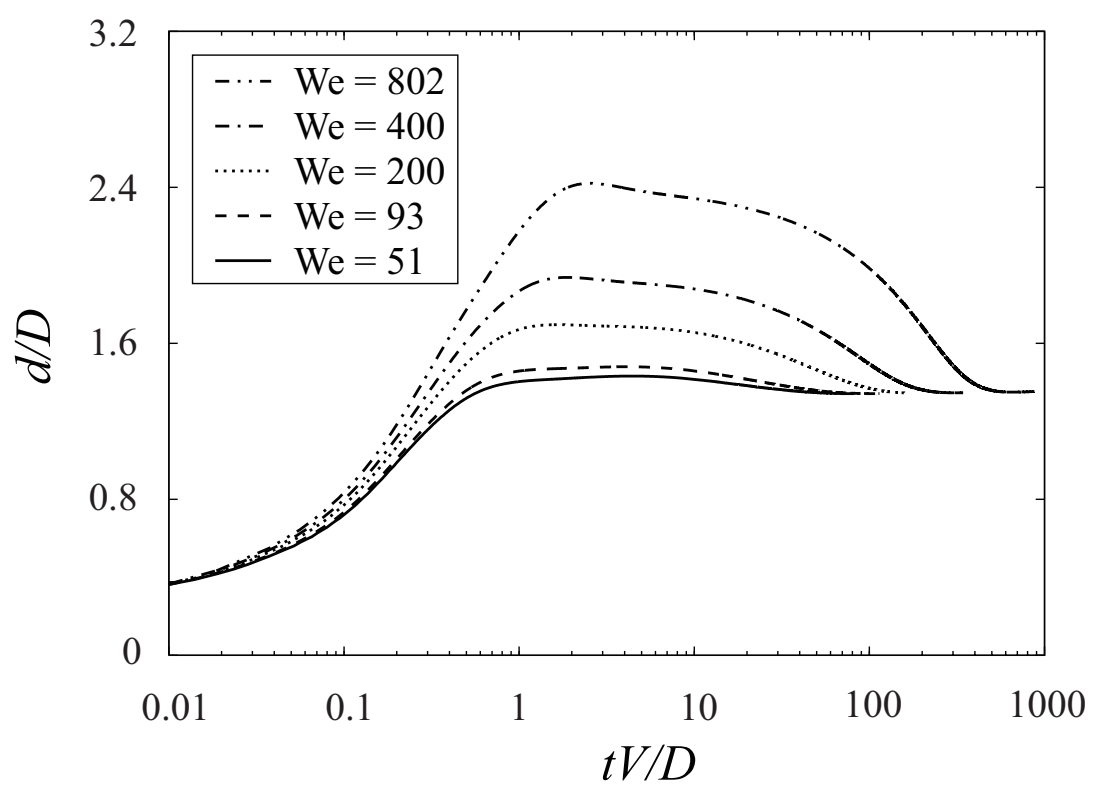

Figure 6: Time variation of $d / D$ for various Weber numbers. 


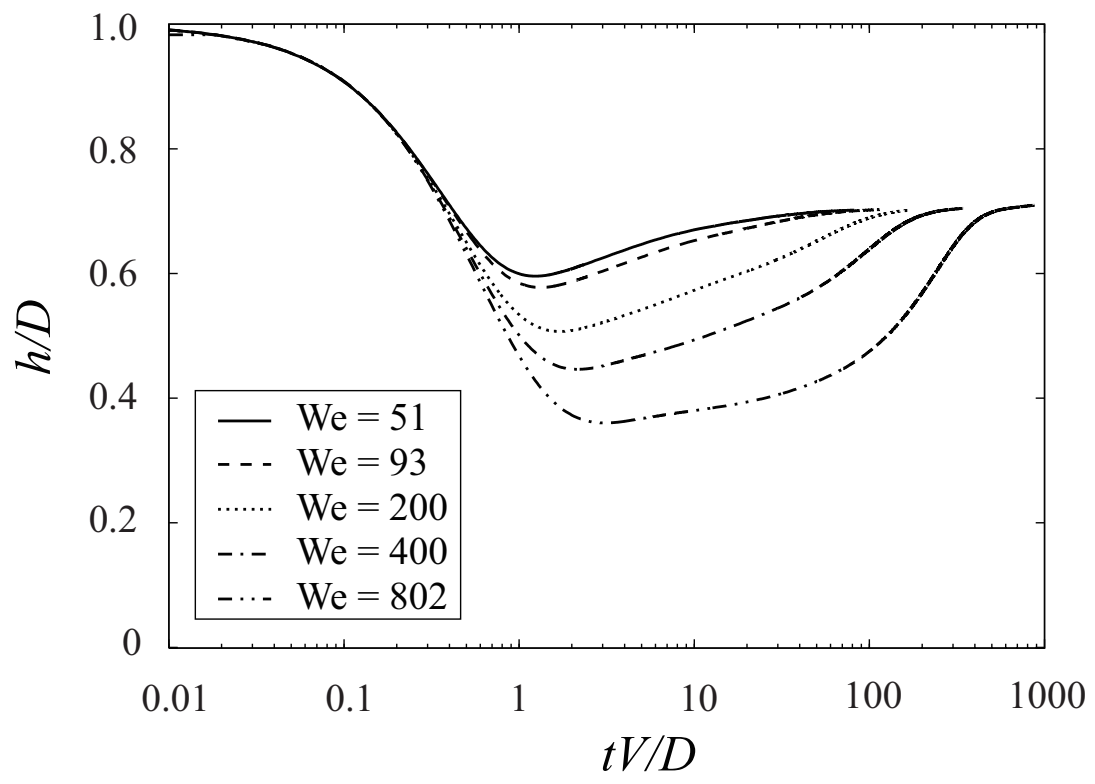

Figure 7: Time variation of $h / D$ for various Weber numbers. 


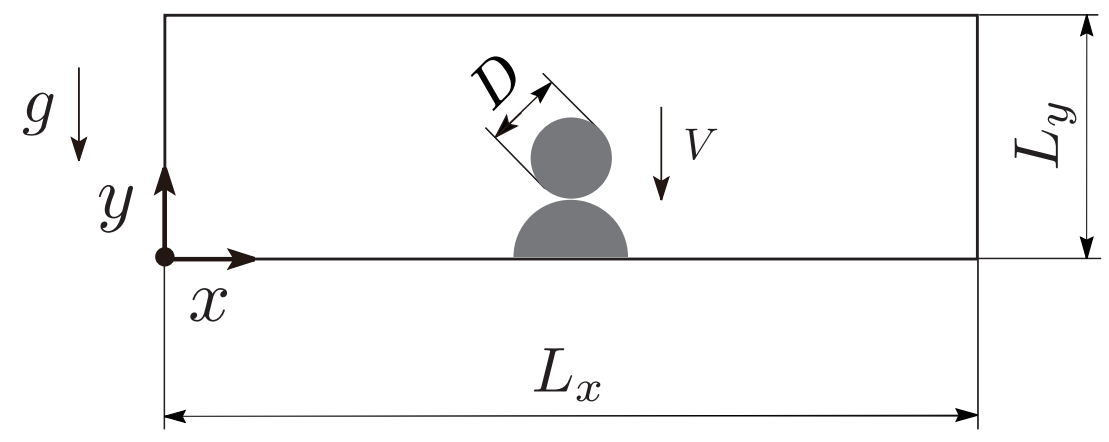

Figure 8: Computational domain of collision of droplets on solid surface. The stationary droplet on the surface is in equilibrium. 


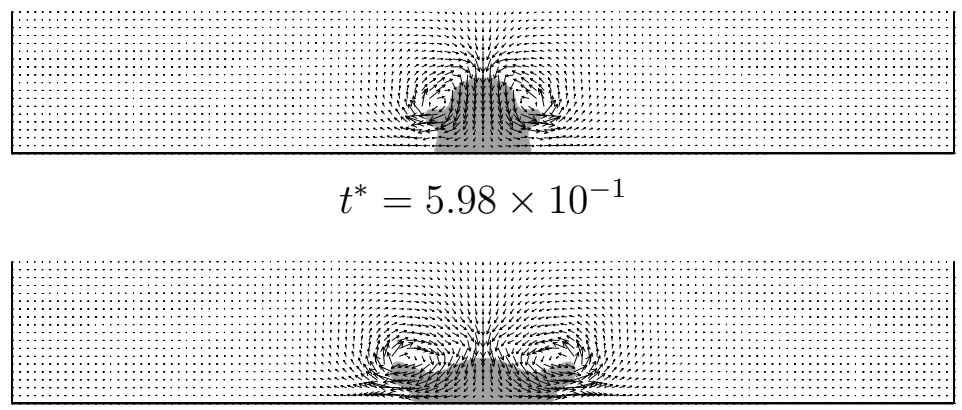

$t^{*}=1.26$

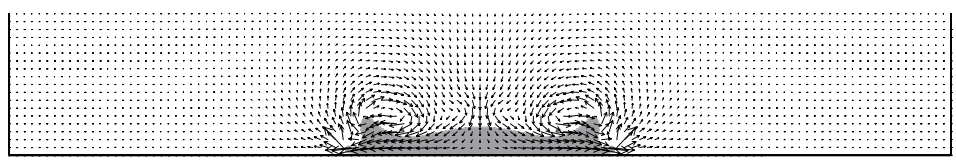

$t^{*}=1.93$

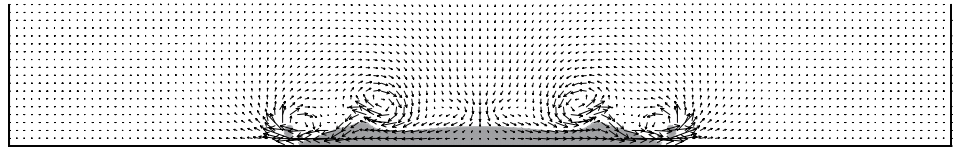

$t^{*}=2.59$

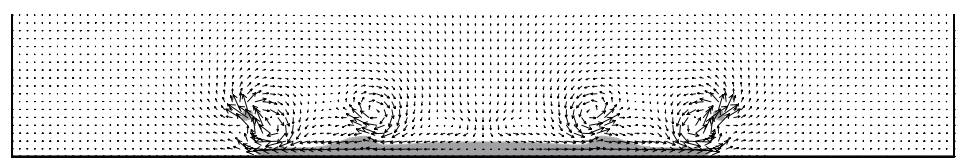

$t^{*}=3.25$

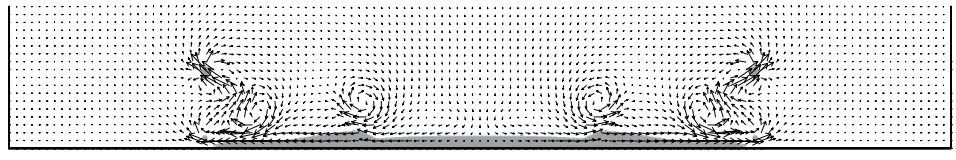

$t^{*}=3.92$

Figure 9: Time evolution of droplet shape and velocity vectors for $W e=50$ and $\theta_{\mathrm{S}}=\pi / 2$ $\left(t^{*}=t V / D\right)$. The lower half of the whole domain $\left(0 \leq y \leq L_{y} / 2\right)$ is shown. 


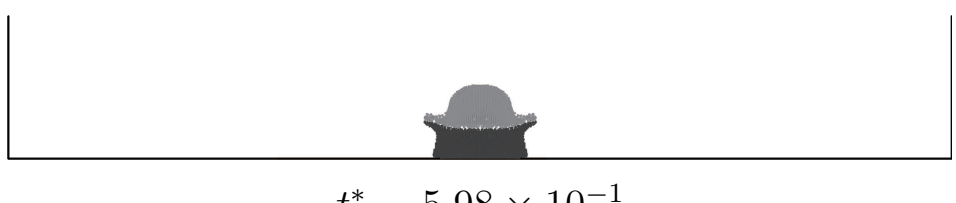

$t^{*}=5.98 \times 10^{-1}$

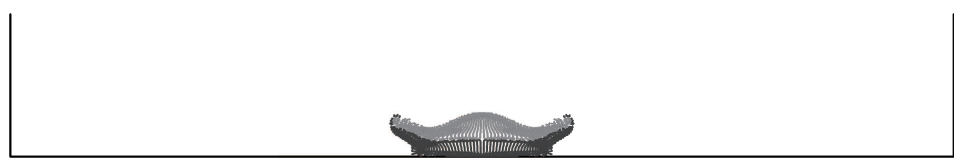

$t^{*}=1.26$

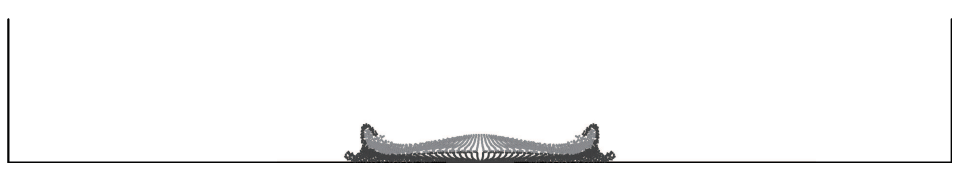

$t^{*}=1.93$

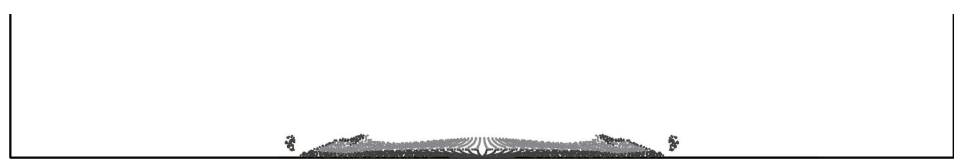

$t^{*}=2.59$

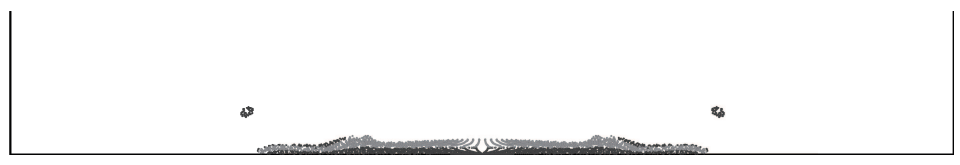

$t^{*}=3.25$

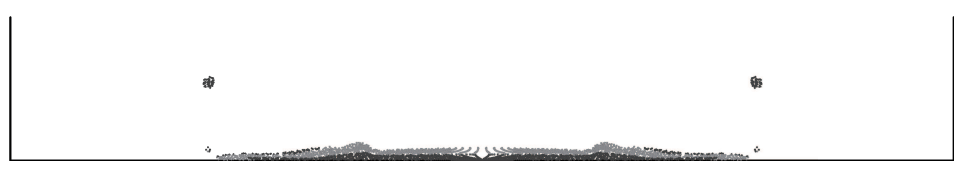

$t^{*}=3.92$

Figure 10: Time evolution of tracer particles for $W e=50$ and $\theta_{\mathrm{S}}=\pi / 2\left(t^{*}=t V / D\right)$. The lower half of the whole domain $\left(0 \leq y \leq L_{y} / 2\right)$ is shown. 


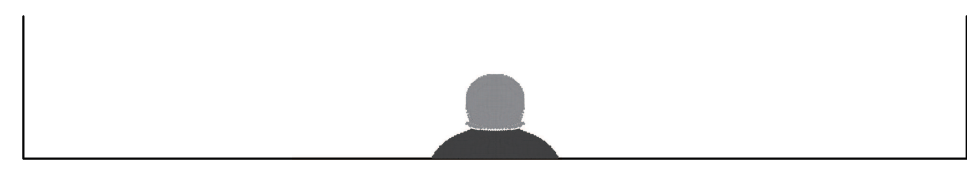

$$
t^{*}=2.06 \times 10^{-1}
$$

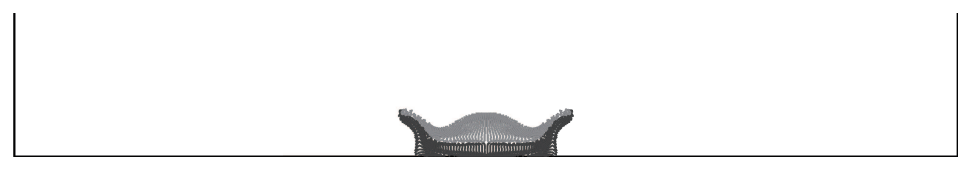

$$
t^{*}=1.00
$$

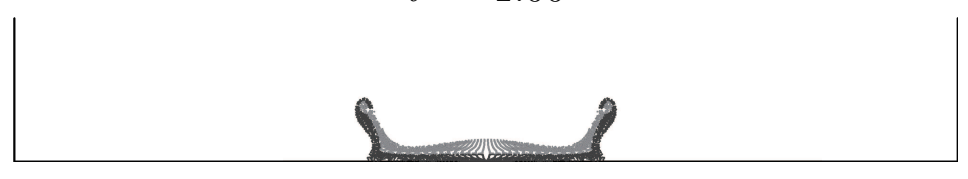

$t^{*}=2.01$
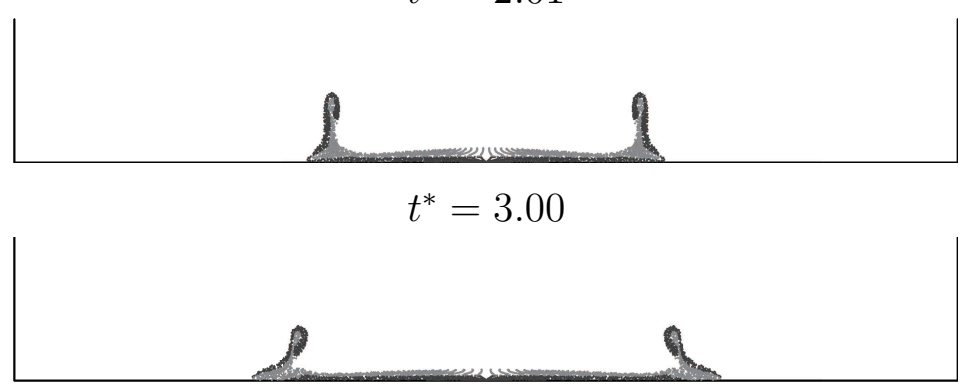

$t^{*}=4.00$

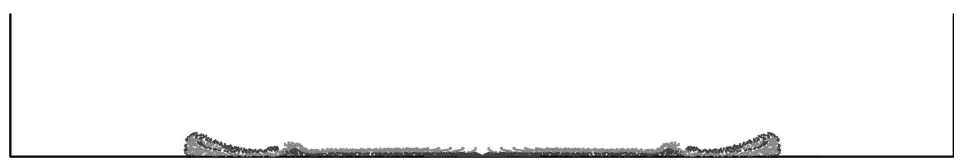

$t^{*}=5.50$

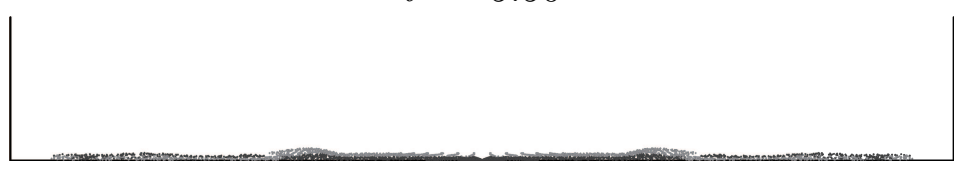

$t^{*}=7.50$

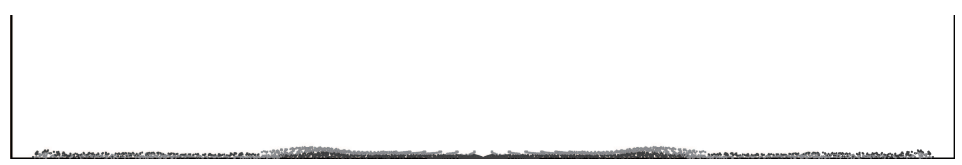

$$
t^{*}=8.63
$$

Figure 11: Time evolution of tracer particles for $W e=50$ and $\theta_{\mathrm{S}}=\pi / 3\left(t^{*}=t V / D\right)$. The lower half of the whole domain $\left(0 \leq y \leq L_{y} / 2\right)$ is shown. 


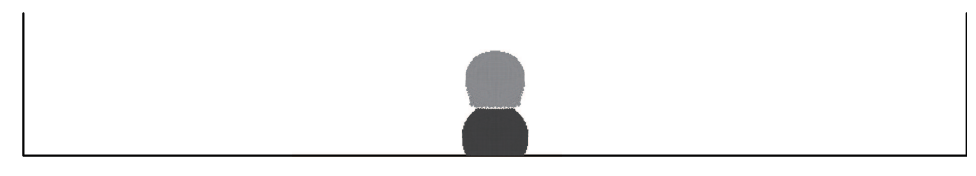

$t^{*}=2.06 \times 10^{-1}$
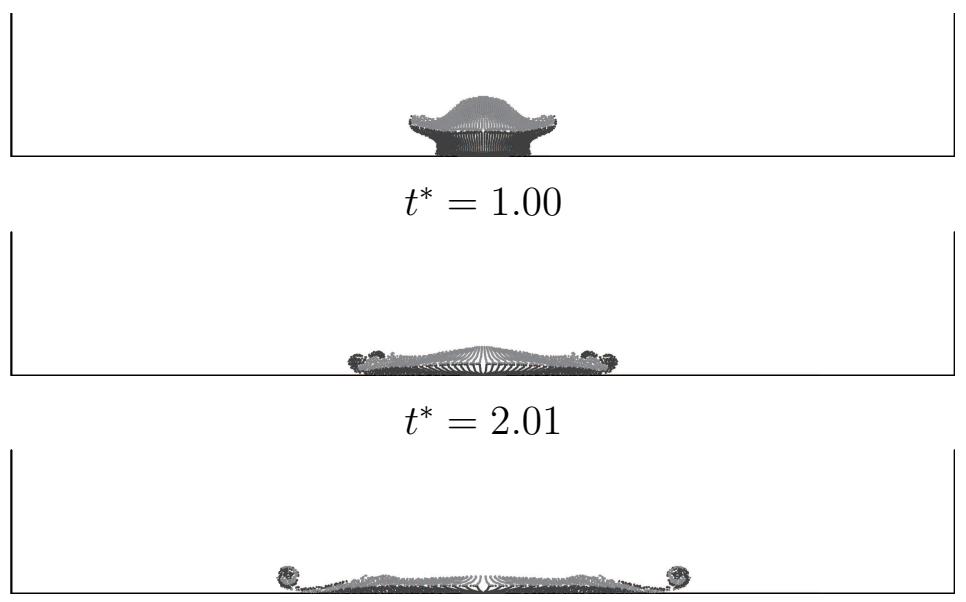

$t^{*}=3.00$

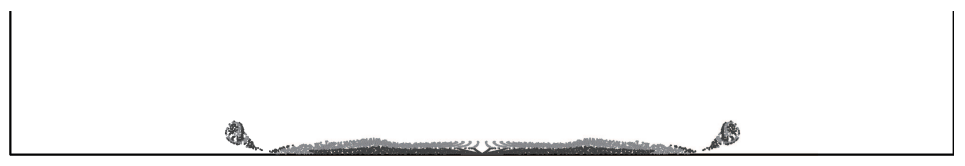

$t^{*}=4.00$

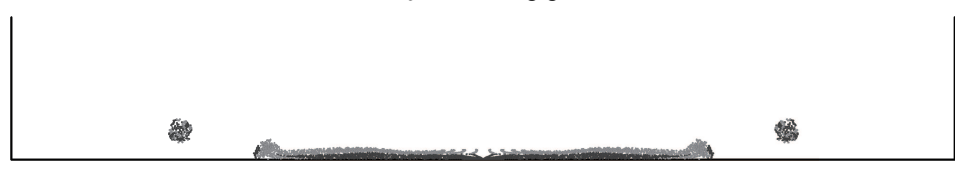

$t^{*}=5.50$

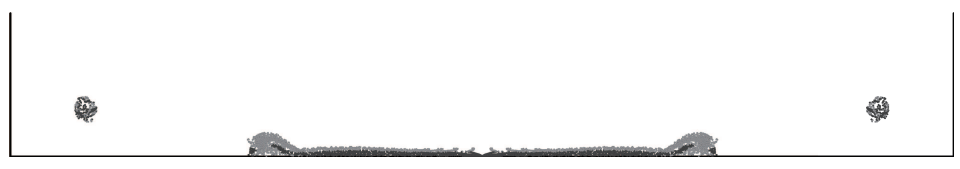

$t^{*}=7.50$

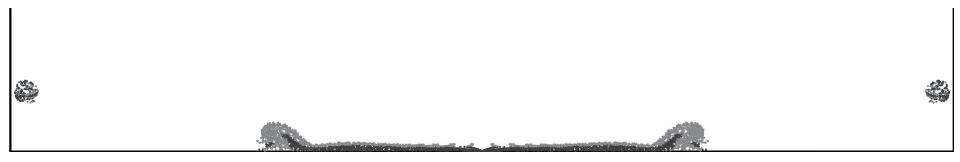

$t^{*}=8.63$

Figure 12: Time evolution of tracer particles for $W e=50$ and $\theta_{\mathrm{S}}=2 \pi / 3\left(t^{*}=t V / D\right)$. The lower half of the whole domain $\left(0 \leq y \leq L_{y} / 2\right)$ is shown. 


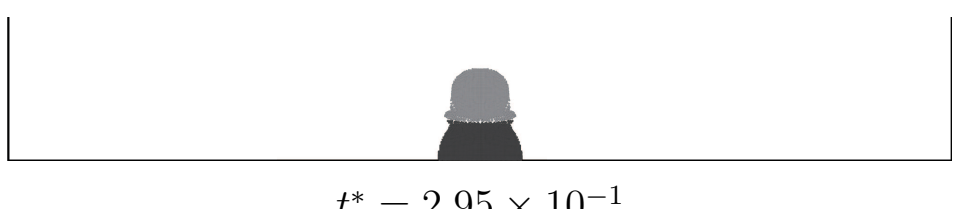

$t^{*}=2.95 \times 10^{-1}$

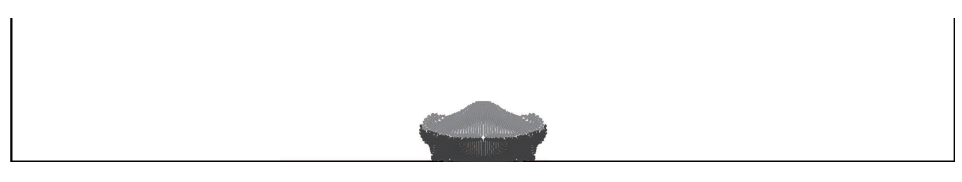

$$
t^{*}=8.20 \times 10^{-1}
$$

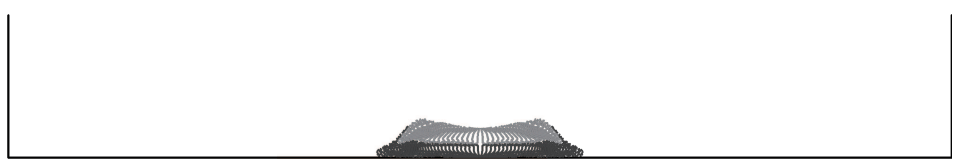

$t^{*}=1.36$

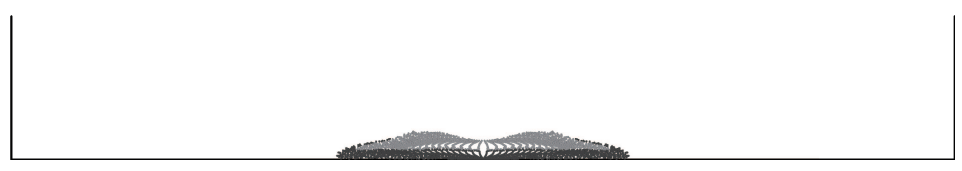

$t^{*}=1.89$

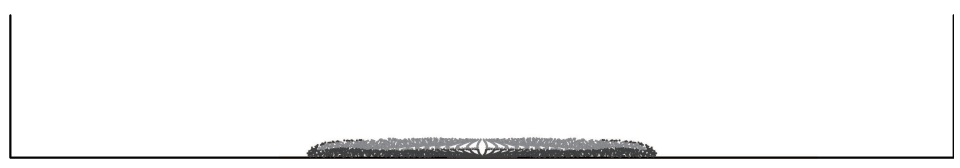

$t^{*}=2.43$

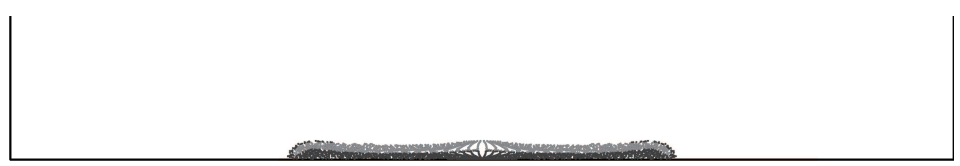

$t^{*}=2.97$

Figure 13: Time evolution of tracer particles for $W e=10$ and $\theta_{\mathrm{S}}=\pi / 2\left(t^{*}=t V / D\right)$. The lower half of the whole domain $\left(0 \leq y \leq L_{y} / 2\right)$ is shown. 


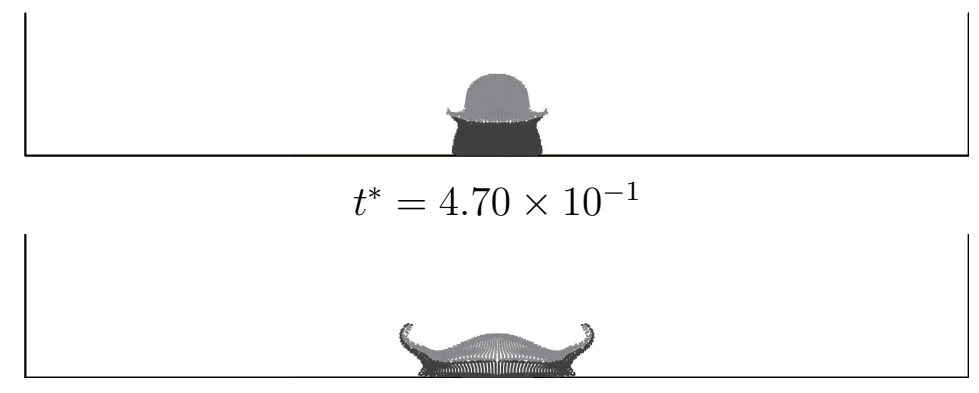

$t^{*}=1.32$

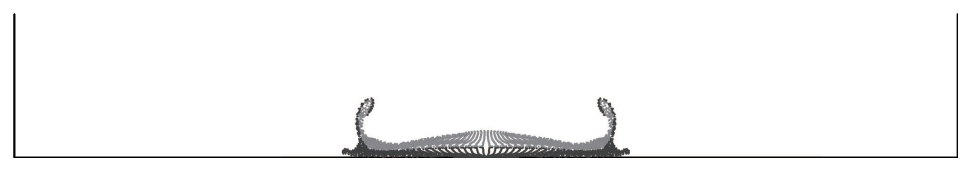

$t^{*}=2.16$

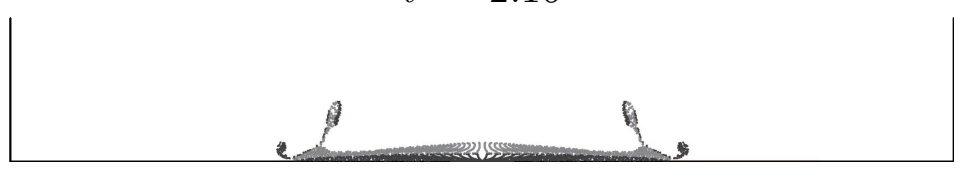

$t^{*}=3.01$

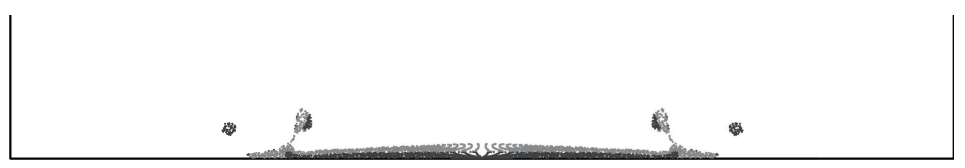

$t^{*}=3.85$

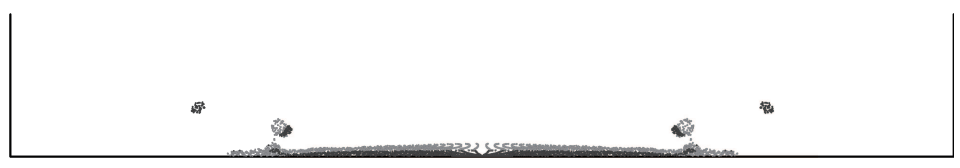

$t^{*}=4.42$

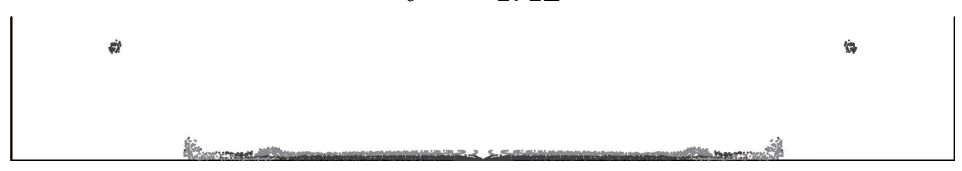

$t^{*}=6.11$

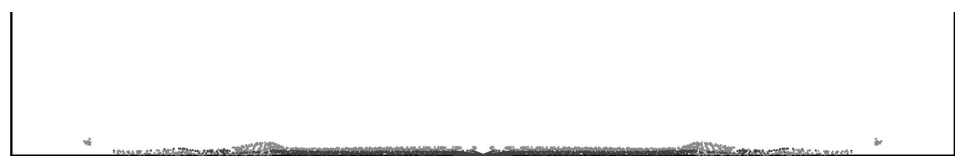

$t^{*}=7.52$

Figure 14: Time evolution of tracer particles for $W e=100$ and $\theta_{\mathrm{S}}=\pi / 2\left(t^{*}=t V / D\right)$. The lower half of the whole domain $\left(0 \leq y \leq L_{y} / 2\right)$ is shown. 


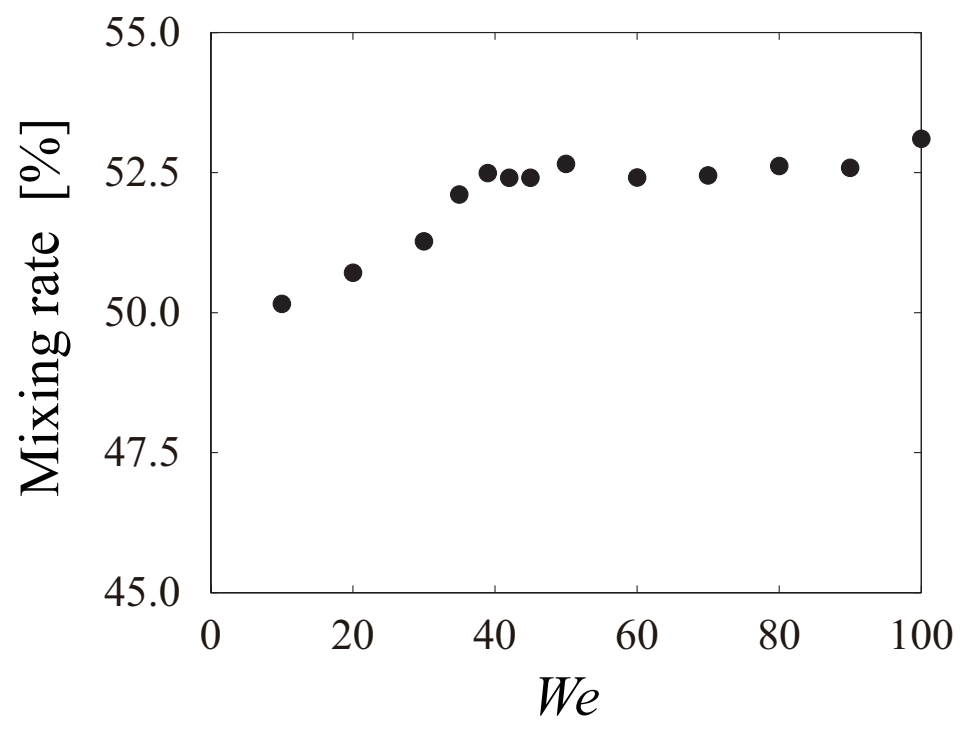

Figure 15: Mixing rate of primary droplet versus Weber number. 